\title{
Coulomb drag: a probe of electron interactions in coupled quantum wells
}

\author{
Jauho, Antti-Pekka
}

Published in:

Proceedings of the International Semiconductor Conference

Link to article, DOI:

10.1109/SMICND.1996.557301

Publication date:

1996

Document Version

Publisher's PDF, also known as Version of record

Link back to DTU Orbit

Citation (APA):

Jauho, A-P. (1996). Coulomb drag: a probe of electron interactions in coupled quantum wells. In Proceedings of the International Semiconductor Conference (Vol. Volume 1, pp. 21-30). IEEE.

https://doi.org/10.1109/SMICND.1996.557301

\section{General rights}

Copyright and moral rights for the publications made accessible in the public portal are retained by the authors and/or other copyright owners and it is a condition of accessing publications that users recognise and abide by the legal requirements associated with these rights.

- Users may download and print one copy of any publication from the public portal for the purpose of private study or research.

- You may not further distribute the material or use it for any profit-making activity or commercial gain

- You may freely distribute the URL identifying the publication in the public portal

If you believe that this document breaches copyright please contact us providing details, and we will remove access to the work immediately and investigate your claim 
INVITED PAPER

\title{
COULOMB DRAG: A PROBE OF ELECTRON INTERACTIONS IN COUPLED QUANTUM WELLS
}

\author{
ANTTI-PEKKA JAUHO \\ Mikroelektronik Centret \\ Technical University of Denmark, Bldg. 345east \\ DK-2800 Lyngby, Denmark
}

\section{Introduction}

As semiconductor devices shrink in size and in dimensionality, interactions between charge carriers become more and more important. There is a simple physical reason behind this behavior: fewer carriers lead to less effective screening, and hence more stronger effective interactions. A point in case are one-dimensional systems (quantum wires): there electron-electron interactions may lead to a behavior, which is qualitatively different from the standard Fermi liquid picture (Luttinger liquids). Electron-electron interactions also play a central role in the fractional quantum Hall effect, which displays an extremely rich physical behavior, and remains a very active area for research. Thus there is a clear need for a better understanding of electron-electron interactions in dimensionally reduced semiconductor structures.

A standard method of studying the properties of semiconductor samples is to perform transport measurements, with or without magnetic field. Electron-electron interactions, however, often affect transport measurements only indirectly, and are often masked by other mechanisms. This difficulty can be overcome by studying double (or multiple) layer systems, as the following argument shows. Consider two systems containing mobile charge carriers so close to each other that the charges in the two respective subsystems feel the Coulomb forces originating from the other subsystem, and yet so far from each other that direct charge transfer, for example by tunneling, between the two systems is not possible. Experimental realizations of such systems are, for example, Coulomb coupled double quantum well systems $[1,2]$, arrangements where a $3 \mathrm{D}$ system is close to a $2 \mathrm{D}$ system [3], or two nearby quantum wires. A scattering event between a carrier in one 


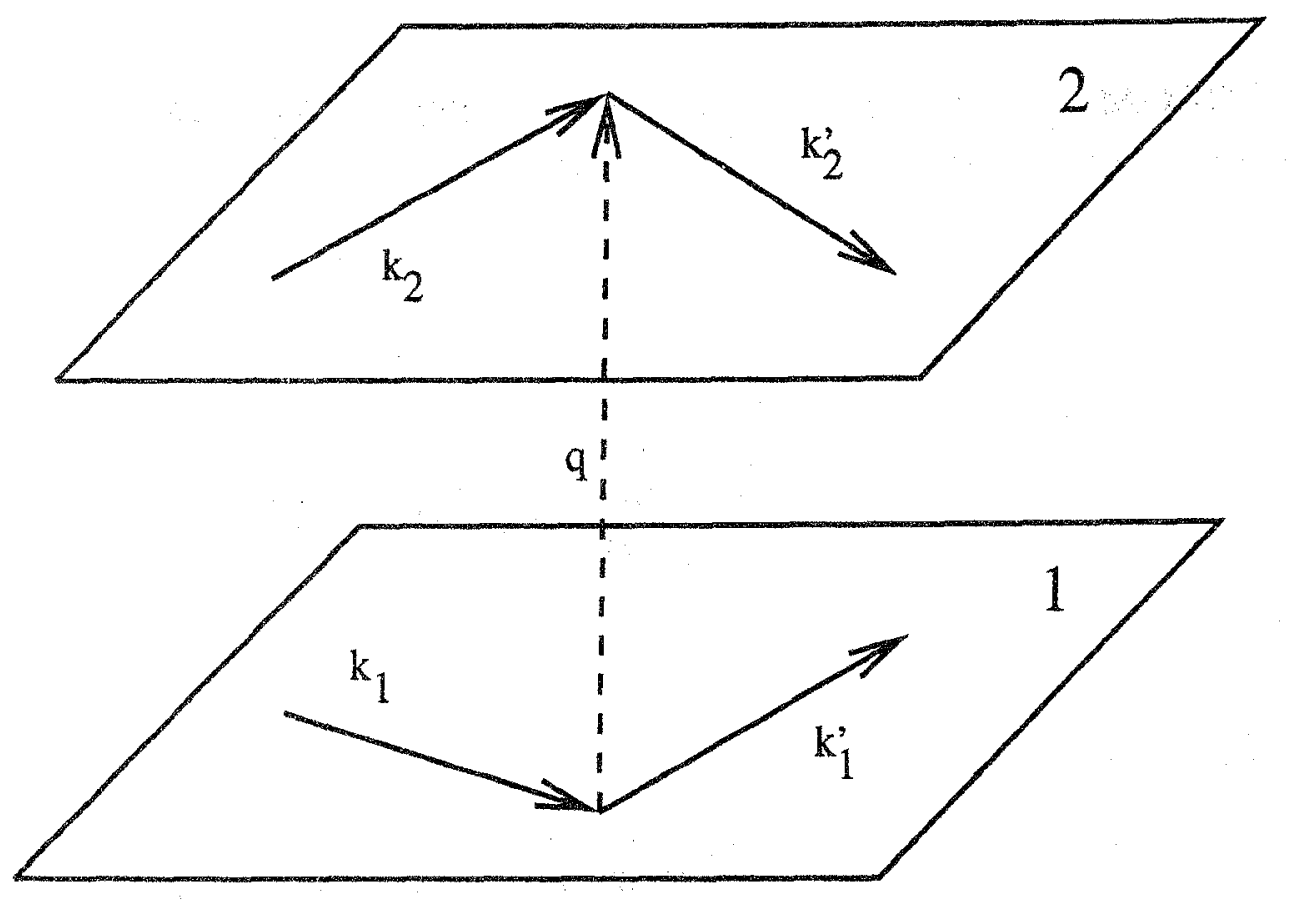

Figure 1. Coulomb drag geometry. A carrier in the drive layer (" 1 ") scatters from state $k_{1}$ to a state $k_{1}^{\prime} \equiv k_{1}-q$ transferring momentum $q$ to the probe layer ("2"), where a carrier scatters from state $k_{2}$ to $k_{2}^{\prime} \equiv k_{2}+q$. In general, the scattering probability may also depend on the transferred energy, $\Delta E=\epsilon\left(\mathbf{k}_{1}^{0}\right)-\epsilon\left(\mathbf{k}_{1}\right)$.

system and a carrier in the other system leads to momentum transfer between the two subsystems (see Fig. 1). Thus, if a current is driven through one of the systems (henceforth the driven system is denoted as layer 1), then an induced current is dragged in the other subsystem (layer 2). Alternatively, if no current is allowed to flow in layer 2 , a voltage is induced. Due to momentum conservation the two particle number currents flow in the same direction. Since the mechanism for the Caulomb drag is due to carrier-carrier scattering the drag current is proportional to the square of the effective interaction between the subsystems. The available phase space for electron-electron scattering tends to zero at low temperatures, and consequently one would expect Coulomb drag to decrease with decreasing temperature. This expectation follows from the Fermi Golden Rule expression for the electron-electron scattering rate, which can qualitatively be written as

$\frac{1}{\tau\left(\mathbf{k}_{1}\right)} \propto \sum_{\mathbf{k}_{1}^{\prime} \mathbf{k}_{2} \mathbf{k}_{2}^{\prime}}|W|^{2} f_{1} f_{2}\left(1-f_{1^{\prime}}\right)\left(1-f_{2^{\prime}}\right) \delta\left(\epsilon_{1}+\epsilon_{2}-\epsilon_{1^{\prime}}-\epsilon_{2^{\prime}}\right) \delta_{\mathbf{k}_{1}+\mathbf{k}_{2}, \mathbf{k}_{1}^{\prime}+\mathbf{k}_{2}^{\prime}}$ 
At low temperatures, the two Pauli factors lead to a $T^{2}$-dependence, and this behavior is approximately seen in experiments [1]. Note, however, that there are small, but important deviations from the simple $T^{2}$-law; these deviations have been the topic of much recent interest $[1,4]$, and we shall comment them below.

The possibility for Coulomb drag was realized already long ago $[5,6]$, and the recent experimental advances $[1,2,3]$ have brought about a flurry of theoretical works. A number of different theoretical approaches has been proposed. These include (i) calculations based on the Boltzmann equation $[1,7]$, (ii) the memory function approach of Ref.[8], (iii) the momentum balance equation method [4], and (iv) diagrammatic linear response (Kubo formula) approaches $[9,10]$. In the present review we shall discuss two of these approaches: the semiclassical Boltzmann equation approach [7], and the Kubo formula [9]. The Boltzmann approach has the advantage of being quite transparent, while the Kubo approach is needed if one wishes to address effects of quantum mechanical origin, such as weak localization or quantizing magnetic fields. What makes these theories particularly interesting is that they lead to definite predictions of new phenomena, that have not yet been seen experimentally.

\section{Boltzmann equation approach for Coulomb drag}

The central quantity, directly relevant to experiment, is the momentum relaxation rate $1 / \tau_{D}$, which can also expressed as a transresistivity, $\rho_{21}=$ $m /\left(n_{2} e^{2} \tau_{D}\right)$ (here $n_{2}$ is the carrier density in the drag layer). The momentum relaxation rate is determined by setting up Coulomb coupled Boltzmann equations for the two layers (the intra-layer scattering mechanisms are described with a relaxation time), and the linearized form of these coupled Boltzmann equations can be used to derive a balance equation between the induced electric field and the drag due to the drive current. The calculations are described in detail in Refs. $[7,11]$, and here we just state the final result, and interpret it physically. The transresistivity is found to be

$$
\rho_{21}^{\beta \alpha}==\frac{\hbar^{2}}{2 \pi e_{1} e_{2} n_{1} n_{2} k_{B} T} \int \frac{d \mathrm{q}}{(2 \pi)^{2}} \int_{0}^{\infty} d \omega \frac{\left.\left|W_{12}(q, \omega)\right|^{2} F_{1}^{\alpha}(\mathbf{q}, \omega)\right] F_{2}^{\beta}(\mathrm{q}, \omega)}{\sinh ^{2}\left(\hbar \omega /\left[2 k_{B} T\right]\right)}
$$

Here, $\{\alpha \beta\}$ label the Cartesian coordinates, $W_{12}(q, \omega)$ is the effective $d y$ namically screened interaction between the carriers in the two layers, and we have defined the function

$$
\begin{aligned}
F^{\alpha}(\mathbf{q}, \omega)= & \frac{2 \pi e}{\hbar \mu_{t}} \int \frac{d \mathbf{k}}{(2 \pi)^{2}}\left[f^{0}(\mathbf{k})-f^{0}(\mathbf{k}+\mathbf{q})\right] \delta(\epsilon(\mathbf{k})-\epsilon(\mathbf{k}+\mathbf{q})-\hbar \omega) \\
& \times\left[v^{\alpha}(\mathbf{k}+\mathbf{q}) \tau(\mathbf{k}+\mathbf{q})-v^{\alpha}(\mathbf{k}) \tau(\mathbf{k})\right]
\end{aligned}
$$




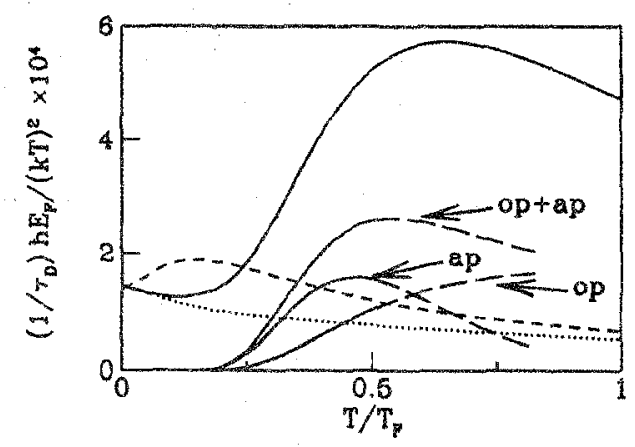

Figure 2. Temperature dependence of the drag rate scaled by $T^{2}$. The full bold curve corresponds to calculations using the finite- $T$ form of $\chi(q, \omega)$, the dotted curve to using the $T=0$ form of $\chi$, and the short-dashed curve is based on the static screening approximation. Also shown are the plasmon-pole approximation estimates for the acoustic plasmon (ap) and optic plasmon (op) contributions to $T_{D}^{-1}$, and the sum of the two $(o p+a p)$. For $T \geq 0.6 T_{F}$, this approximation becomes less reliable due to large Landau damping of the modes, and hence we have plotted the results with dashed lines.

where $\mu_{t}$ is the mobility, and $\tau(\mathrm{k})$ is the transport relaxation time. This result can be understood physically as follows. In the limit of a constant transport relaxation time, one finds that $F^{\alpha}(\mathbf{q}, \omega) \rightarrow q^{\alpha} \operatorname{Im} \chi(\mathbf{q}, \omega)$, where $\chi$ is the polarization function. In RPA, for example, $\chi$ is given by the Lindhard function. Thus, the transresistivity can be approximately written as $\rho_{12} \propto$ $\int d q \int d \omega q^{2} \times W^{2} \times \operatorname{Im} \chi^{2} / \sinh ^{2}\left(\frac{\hbar \omega}{2 k_{B} T}\right)$, where the different factors have the following interpretation: (i) $q^{2}$ - momentum transfer; (ii) $W^{2}$ - effective interaction; and (ii) $\operatorname{Im} \chi$ combined with the sinh-factor - phase-space (this term can be linked, via the fluctuation dissipation theorem, to the structure factor). Several authors have performed numerical calculations based on Eq.(2), and they are summarized in Fig. 2. The following features are noteworthy. (i) The overall drag rate, when scaled by $T^{2}$, is not a constant, as the simple arguments presented in the introduction would suggest. (ii) When compared to the experiments of Ref.[1], one sees that the calculated low-temperature $1 / \tau_{D}\left(T<6 K\right.$, or, referring to Fig. $\left.2, T<T_{F} / 10\right)$ is about one-half of the experimentally observed rate. The probable cause for this underestimation are virtual phonon-mediated interactions, which are not included in the calculations of Fig. $2[1,4]$. Note, however, that the form of Eq.(2) is quite general: the particular form of the effective interaction has not yet been specified. (iii) More importantly, the drag rate is a very sensitive function of the screening model; thus one must use a fairly sophisticated polarization function (neither zero-temperature nor 
static response functions are sufficient), and at low densities even RPA may fail [13]. (iv) Finally, Fig. 2 contains a striking prediction [11, 12]: the drag rate is strongly enhanced at about $T=0.5 T_{F}$. The enhancement is due to plasmons; thus Coulomb drag offers a means of directly probing the collective excitations of the double-layer system. To our knowledge, this enhancement has not yet been experimentally verified.

If one wishes to study quantum mechanical effects, such as weak localization, a more refined theoretical method is needed, and we describe such an approach in the next section.

\section{Kubo formula approach to Coulomb drag}

The Kubo formula [14] expresses the conductivity tensor in terms of the retarded current-current correlation function,

$$
\sigma_{i j}^{\alpha \beta}\left(\mathbf{x}-\mathbf{x}^{\prime} ; \Omega\right)=\frac{i e^{2}}{\Omega} \Pi_{i j}^{\alpha \beta, r}\left(\mathbf{x}-\mathbf{x}^{\prime}, \Omega\right)+\frac{i e^{2}}{m \Omega} \delta\left(\mathbf{x}-\mathbf{x}^{\prime}\right) \delta_{i j} \delta_{\alpha \beta} \rho_{j}(\mathbf{x}),
$$

where

$$
\Pi_{i j}^{\alpha \beta, r}\left(\mathbf{x}-\mathbf{x}^{\prime}, t-t^{\prime}\right)=-i \theta\left(t-t^{\prime}\right)\left\langle\left[j_{i}^{\alpha}(\mathbf{x}, t), j_{j}^{\alpha}\left(\mathbf{x}^{\prime}, t^{\prime}\right)\right]\right\rangle .
$$

Here $\{i j\}$ indicate the subsystem, $\Omega$ is the external frequency, $\rho_{i}(\mathbf{x})$ is the particle density in subsystem $i$, and $\mathbf{j}(\mathbf{x}, t)$ is the particle current operator.

The evaluation of $\sigma_{21}^{\alpha \beta}$ (which essentially gives $\rho_{21}$ ) proceeds through the following steps. (i) One uses the Matsubara formalism [14] to calculate the (imaginary-)time-ordered correlation function corresponding to Eq.(5); (ii) The transconductivity is expanded in powers of the interaction $H_{12}$ between the sybsystems,

$$
H_{12}=\int d \mathbf{r}_{1} \int d \mathbf{r}_{2} \rho_{1}\left(\mathbf{r}_{1}\right) U_{12}\left(\mathbf{r}_{1}-\mathbf{r}_{2}\right) \rho_{2}\left(\mathbf{r}_{2}\right),
$$

and, finally, (iii) the imaginary-time correlation function is analytically continued to extract the retarded correlation function. The upshot of the analysis is that the dc transconductivity for uniform systems can be written as

$$
\begin{aligned}
\sigma_{21}^{\alpha \beta(2)}= & \frac{e^{2}}{\hbar}\left(-\frac{1}{2}\right) \frac{1}{\nu} \sum_{\mathbf{q}} \int_{-\infty}^{\infty} \frac{d \omega}{2 \pi}\left|W_{12}(q, \omega)\right|^{2}\left[\partial_{\omega} n_{\mathrm{B}}(\omega)\right] \\
& \times \Delta_{1}^{\alpha}\left(\mathbf{q}, \mathbf{q} ; \omega^{+}, \omega^{-}\right) \Delta_{2}^{\beta}\left(-\mathbf{q},-\mathbf{q} ;-\omega^{-},-\omega^{+}\right) .
\end{aligned}
$$

where $n_{\mathrm{B}}(\omega)$ is the Bose function, and the three-particle correlation function $\Delta$ is the analytic continuation of the Fourier-transform of

$$
\Delta_{i}\left(\mathbf{x}, \tau, \mathbf{x}^{\prime}, \tau^{\prime}, \mathbf{x}^{\prime \prime}, \tau^{\prime \prime}\right)=-\left\langle T_{\tau}\left\{j_{i}^{\alpha}(\mathbf{x}, \tau) \rho_{i}\left(\mathbf{x}^{\prime}, \tau^{\prime}\right) \rho_{i}\left(\mathbf{x}^{\prime \prime}, \tau^{\prime \prime}\right)\right\rangle .\right.
$$




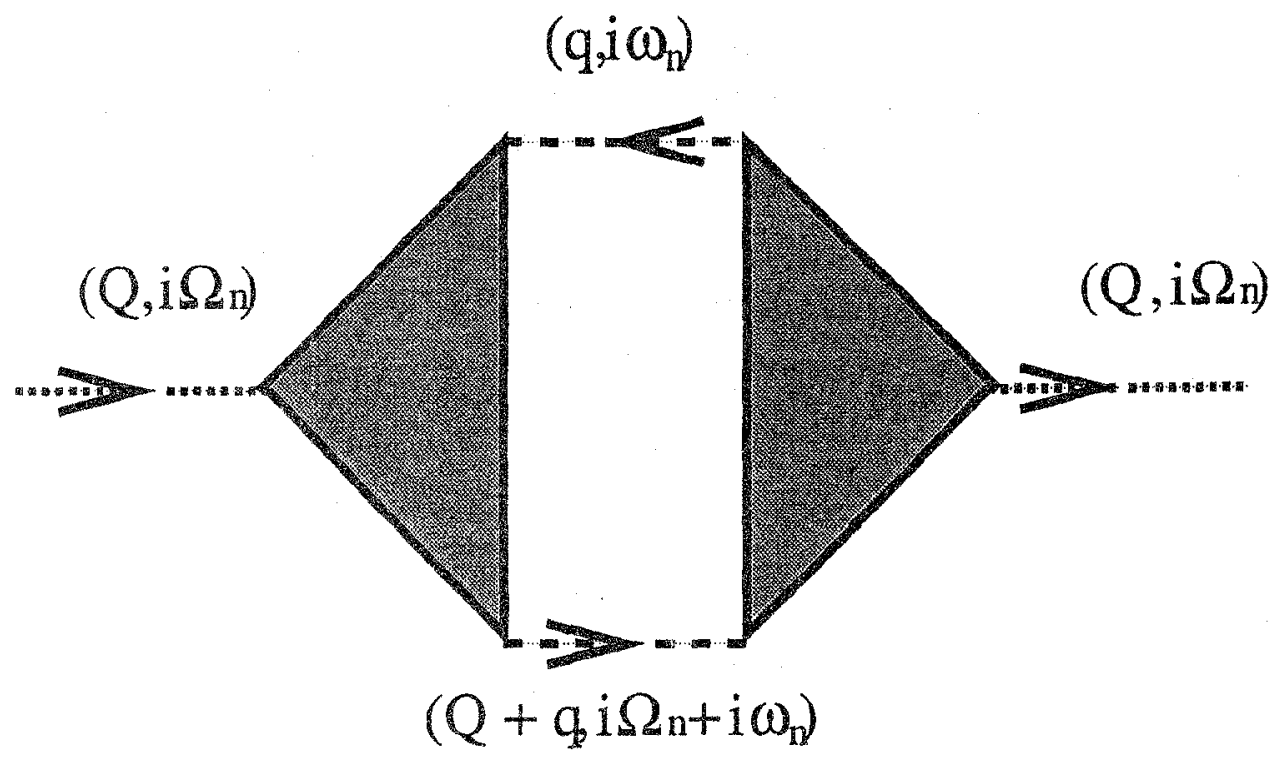

Figure 3. Diagram corresponding to the current-current correlation function to second order in the interlayer Coulomb interaction. The shaded triangles correspond to the three-body correlation functions $\Delta_{1,2}$ given in Eq.(7), the dashed lines to the interaction, the dotted lines to the external current operators, and the arrowheads indicate the direction of momentum and energy transfer.

Fig. 3 shows the diagrammatic representation of the transconductivity; similar fluctuation diagrams are known from superconductivity $[15,16]$, and from the microscopic theory of van der Waals interactions [17, 18]. It can be shown that Eq.(7) reproduces the Boltzmann results in the appropriate limit [9]. Further, Ref.[9] has shown that the weak localization corrections are small, and Ref.[10] has discussed applications to superconducting drag. In the following section we outline the calculation in a quantizing magnetic field.

\section{Coulomb drag in a magnetic field}

The combination of electron-electron interaction and strong magnetic fields in two-dimensional electron gases has provided an exciting venue of research for both experimentalists and theorists over the past few decades [19]. One well-known example of this is the fractional quantum Hall effect, where the physics is determined by the subtle interplay between interactions and the large density of states caused by all the electrons being confined to the lowest Landau level. Thus, phenomena involving interelectron interactions in a $B$-field often produce surprising and interesting results.

Since Coulomb drag displays rich physics, it is quite natural to ask 


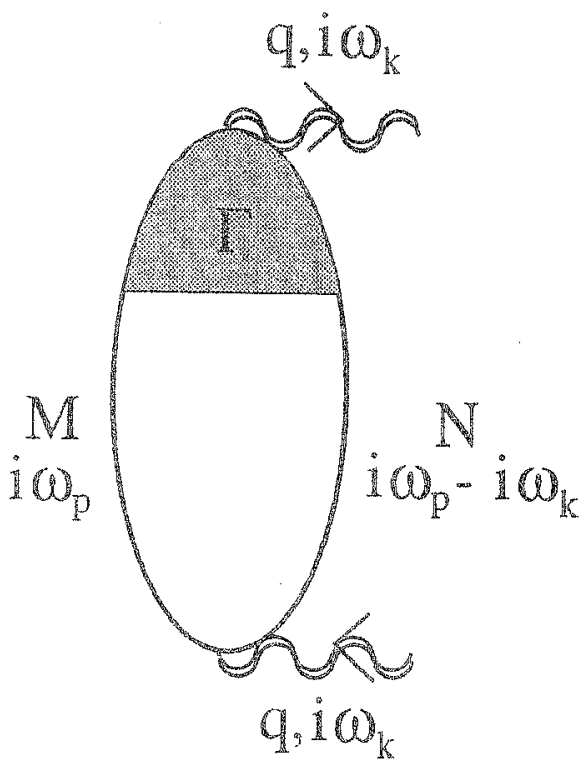

(a)

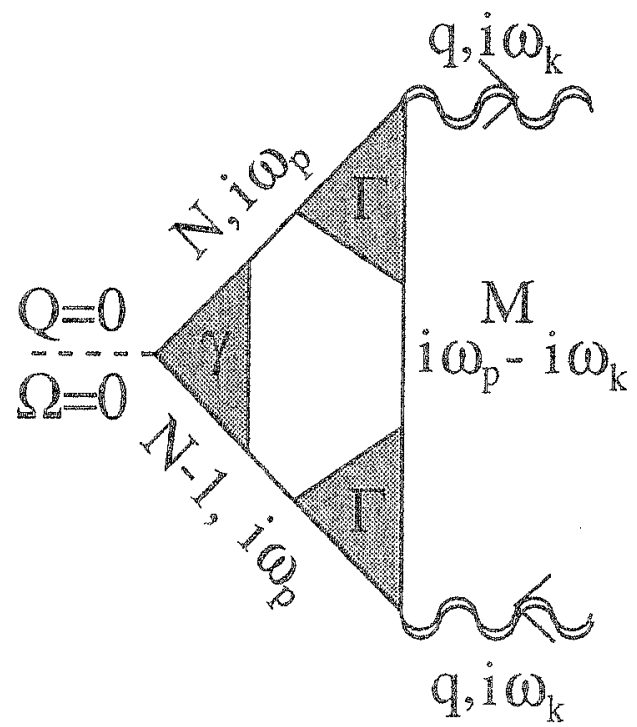

(b)

Figure 4. (a) The diagram corresponding to the density response function $\chi$. (b) The triangle diagram contribution to the 3 -body response function $\Delta$. The $\Gamma$ and $\gamma$ are the charge and current vertices, respectively, and the labels $M$ and $N$ denote the LL's.

whether an applied magnetic field would introduce some further interesting aspects. It turns out, however, that experiments are not easy, and only very recently the first preliminary results have begun to emerge [20]. A similar situation exists on the theoretical side: it is not at all obvious whether standard theories are applicable in the presence of a strong magnetic field. However, the rigorous linear response theory described in the previous section provides an ideal starting point for developing a theory for magnetoCoulomb drag, and we have recently completed such an calculation [21]. In the remainder of this review we briefly sketch some of our central results.

A pure system in a quantizing magnetic field has a singular density of states, and it is necessary to include impurities to avoid unphysical results. In our diagrammatic language this implies that both screening (the "bubble" diagrarn) and the triangle diagram must be evaluated with vertex. functions (see Fig. 4). This leads to quite involved calculations [21]; however, many simplifications occur in the weak-scattering limit $\omega_{c} \tau \gg 1$ (here $\omega_{c}=e B / m$ is the cyclotron frequency and $\tau$ is the impurity scattering time). In particular it is possible to link $\Delta$ with the $\chi(\mathbf{q}, \omega)$ shown in Fig. 

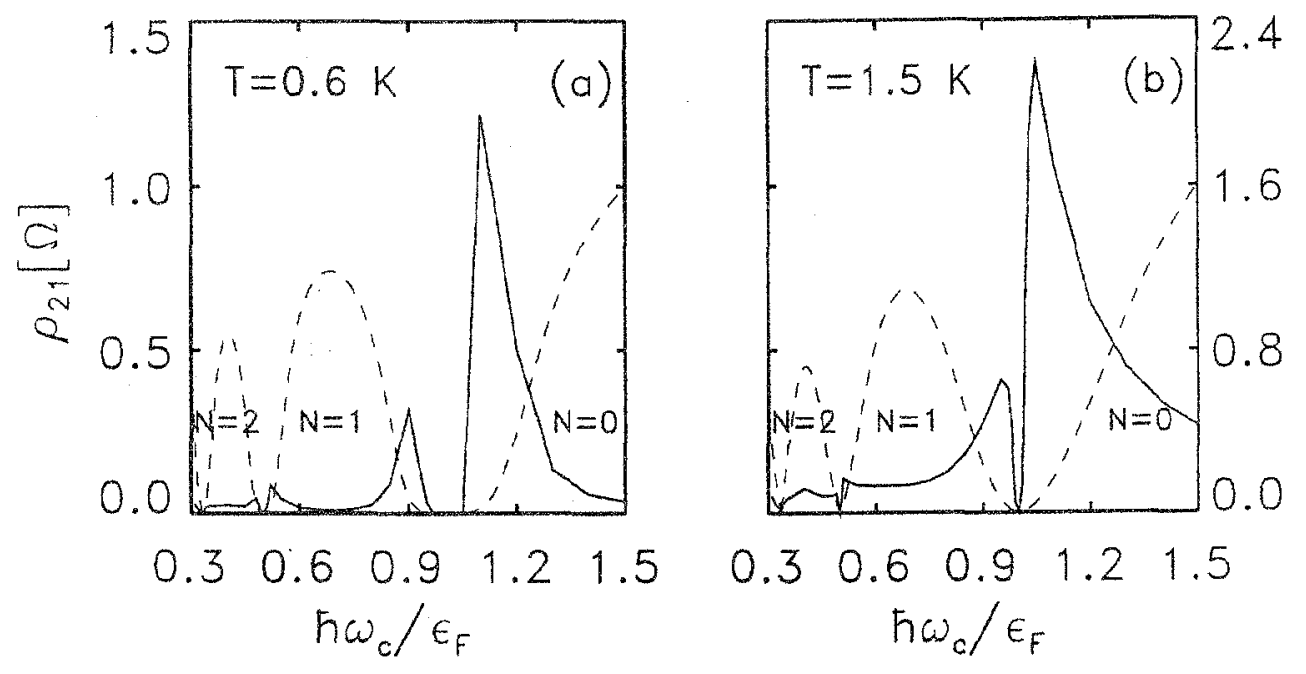

Figure 5. Transresistivity $\rho_{21}$ (solid lines) and and the thermally averaged DOS $g=\partial n / \partial \mu$ (dashed, in arbitrary units) for (a) $T=0.6 \mathrm{~K}$, and (b) $T=1.5 \mathrm{~K}$ as a function of magnetic field in GaAs for density $n=1.5 \times 10^{11} \mathrm{~cm}^{-2}\left(E_{F} / k_{B} \approx 60 \mathrm{~K}\right)$, well separation $d=350 \AA$ and zero well widths. $N$ is the LL index, and $\hbar \omega_{c}=\epsilon_{F}$ corresponds to $B=3.1 \mathrm{~T}$. While the $g(B)$ peaks in the middle of the Landau level, the interiayer coupling is weakest there (due to large screening), pushing the peaks in $\rho_{21}$ towards the edges of the Landau level bands.

$4(\mathrm{a}):$

$$
\Delta\left(\mathbf{q}, \mathbf{q} ; \omega \pm i 0^{+}, \omega \mp i 0^{+}\right)= \pm 2 \hbar^{2} e^{-1} \mathbf{q} \times \mathbf{B} \frac{\operatorname{Im}[\chi(\mathbf{q}, \omega)]}{B^{2}}+\mathcal{O}\left[\left(\omega_{c} \tau\right)^{-1}\right] .
$$

Eqs.(7) and (9) form the basis of our numerical calculations: the response function $\chi$ is found by solving the appropriate vertex equation in the ladder approximation, and $\Delta$, as given by (9), can be used in (7) to get the transconductivity, and hence the transresistivity.

As remarked above, we expect the low-temperature transresistivity for fixed $T$ to be directly proportional to the product of the thermally averaged density of states of both layers, $g_{1}(B) \times g_{2}(B)$, around the chemical potential $\mu$. Since the density of states is strongly enhanced over its zero-field value, one might expect that (i) $\left|\rho_{21}^{x x}\left(\omega_{c} \tau \gg 1\right)\right| \gg\left|\rho_{21}^{x x}(B=0)\right|$, and (ii) $\rho_{21}^{x x}(B)$ should more or less simply reflect the shape of $g_{1}(B) g_{2}(B)$. 
Fig. 5 shows the results of a calculation for $\rho_{21}^{x x}(B)$ for two identical layers at fixed densities. For comparison, we also show $g(b)$. As expected, $\rho_{21}^{x x}$ is very large: approximately 50-100 times larger than at $B=0$. This is in qualitative agreement with recent experimental results [20]. Also, $\rho_{21}^{x x}$ is largest when $\mu$ is in the bands of extended states, and suppressed when it is in between the extended bands. However, the shape of $\rho_{21}^{x x}$ is markedly different from $g^{2}(B)$.

We can understand this surprising behavior as follows. Recall that $\rho_{21}$ also depends on the interlayer coupling, i.e., also on the screening properties of the system. For two-dimensional electron gases the screened interaction varies inversely with $g$ [22]. Therefore, increasing $g(B)$ weakens the interlayer coupling, implying that the terms $g_{1} g_{2}$ and $|W|^{2}$ tend to work in opposition. This results in the following scenario when $B$ is changed. When $\mu$ lies in the region of localized states below a Landau level band, $\rho_{21}$ is very small because very few electrons have sufficient energy to be excited into extended states where they contribute to the drag. As $B$ is increased so that $\mu$ moves into the Landau level band, the density of extended states increases, while the interlayer interaction is strong due to weak screening, resulting in a sharp rise in $\rho_{21}$. However, as the magnetic field is further increased so that $\mu$ moves closer towards the center of the Landau level and the density of states further increases, the screening becomes so effective that it more than compensates for the increase in density of states, leading to a reduction in $\rho_{21}$. This competition of density of states and screening produces the unique shape of $\rho_{21}(B)$.

\section{Conclusions}

We have reviewed recent developments in the theory of Coulomb drag. Our calculations lead to several predictions of effects not yet seen experimentally. We conclude that Coulomb drag, in particular when combined with magnetic fields, is a very versatile tool for directly probing interparticle interactions in dimensionally restricted systems. A further line for research could be the study of quantum wires: there the interactions may lead to even more dramatic effects.

\section{ACKNOWLEDGEMENT}

The work described in this review is performed in collaboration with Henrik Smith, Karsten Flensberg, Martin Bønsager, Ben Yu-Kuang Hu, and Jari Kinaret. The author is grateful to his colleagues for sharing their insights, and for providing a pleasant working atmosphere. 


\section{References}

1. T. J. Gramila et al., Phys. Rev. Lett. 66, 1216 (1991).

2. U. Sivan, P. M. Solomon, and H. Shtrikman, Phys. Rev. Lett. 68, 1196 (1992)

3. B. Laikhtman and P. M. Solomon, Phys. Rev. B 41, 9921 (1990)

4. H. C. Tso and P. Vasilopoulos and F. M. Peeters, Phys. Rev. Lett. 68, 2516 (1992); ibid. 70, 2146 (1993); H. C. Tso and P. Vasilopoulos, Phys. Rev. B 45, 1333 (1992).

5. M. B. Pogrebinskii, Fiz. Tekh. Poluporovdn. 11, 637 (1977) [Sov. Phys. Semicond. $11,372(1977)]$

6. P. J. Price, Physica B 117, 750 (1983)

7. A.-P. Jauho and H. Smith, Phys. Rev. B 47, 4420 (1993).

8. L. Zheng and A. H. MacDonald, Phys. Rev. B 48, 8203 (1993).

9. K. Flensberg, B. Y.-K. Hu, A. P. Jauho, and J. Kinaret, Phys. Rev. B52, 14761

10. A. Kamenev and Y. Oreg, Phys, Rev. B 52, 7516 (1995)

11. K. Flensberg and B. Y..K. Hu, Phys, Rev. B 52, 14796 (1995)

12. K. Flensberg and B. Y-K. Hu, Phys. Rev. Lett 73, 3572 (1994)

13. L. Swierkowski, J. Szymański, and Z. W. Gortel, Phys. Rev. Lett. 74, 3245 (1995)

14. G. D. Mahan, Many-Particle Physics (Plenum Press, New York, 1990).

15. L. G. Aslamazov and A. I. Larkin, Fiz. Tverd. Tela 10, 1104 (1968), [Soviet Physics - Solid State 10, 875 (1968)]

16. K. Maki, Progr. Theor. Phys. (Kyoto) 39, 897 (1968); R. S. Thompson, Phys. Rev. B 1, 327 (1970)

17. K. Rapcewicz and N. W. Ashcroft, Phys, Rev. B 44, 4032 (1991)

18. R. E. Goldstein, G. Parola, and A. P. Smith, J. Chem. Phys. 91, 1843 (1989)

19. See, for example, articles in The Quantum. Hall Effect, 2nd Edition, Eds. R. E. Prange and S. M. Girvin (Springer-Verlag, New York, 1990)

20. N. Hill, University of Cambridge, unpublished experiments. We are grateful for Dr. Hill for informing us about these results.

21. M. Bønsager, K. Flensberg, B. Y.-K. Hu, and A. P. Jauho (unpublished)

22. T. Ando, A. B. Fowler, and F. Stern, Rev. Mod. Phys. 54, 437 (1982) 